\title{
A formalization of one of the main claims of "FTO Obesity Variant Circuitry and Adipocyte Browning in Humans" by Claussnitzer et al. $2015^{1}$
}

\author{
Amelia Joslin \\ Maze Therapeutics, USA \\ E-mail: ajoslin@mazetx.com; ORCID: https://orcid.org/0000-0003-2432-4125 \\ Editor: Cristina-Iulia Bucur (https://orcid.org/0000-0002-7114-6459) \\ Review comments from: Mariya Dimitrova (https://orcid.org/0000-0002-8083-6048); Tobias Kuhn \\ (https://orcid.org/0000-0002-1267-0234); Davide Ceolin (https://orcid.org/0000-0002-3357-9130); Cristina-Iulia Bucur \\ (https://orcid.org/0000-0002-7114-6459)
}

Received 21 June 2021

Accepted 12 November 2021

\begin{abstract}
Claussnitzer et al. claimed in previous work that a regulatory element within the first intron of FTO affects IRX3 and IRX5 expression during early adipogenesis. We present here a formalization of that claim, stating that all things of class "early human adipogenesis" that are in the context of a thing of class "regulatory element within the first intron of FTO" generally have a relation of type "affects" to a thing of class "expression of genes IRX3 and IRX5" in the same context.
\end{abstract}

Keywords: Early human adipogenesis, regulatory element within the first intron of FTO, expression of genes IRX3 and IRX5

\section{Introduction}

Claussnitzer et al. [1] state that "The rs1421085 T-to-C single-nucleotide variant disrupts a conserved motif for the ARID5B repressor, which leads to derepression of a potent preadipocyte enhancer and a doubling of IRX3 and IRX5 expression during early adipocyte differentiation.". We present here a formalization of the main scientific claim from this quote by using a semantic template called the superpattern [2].

\section{Formalization}

Our formalization looks as follows:

\footnotetext{
${ }^{1}$ As RDF/nanopublication: http://purl.org/np/RAxBBJ2WkonyQN1XfdCAOaCi64J_xqgVGeaLjVQow9M88
} 
CONTEXT-CLASS ("in the context of all ..."): SUBJECT-CLASS (“things of type ...”): QUALIFIER:

RELATION-TYPE ("have a relation of type...”): OBJECT-CLASS (“to things of type...”): early human adipogenesis

regulatory element within the first intron of FTO generally

affects

expression of genes IRX3 and IRX5

In the context class we use a new minted class "early human adipogenesis" that is a subclass of "adipogenesis" (Q2824461) from Wikidata. In the subject class, we use a new minted class "regulatory element within the first intron of FTO" that is related to the class "FTO" (Q14912501) and "intron" (Q207551) from Wikidata. In the object class we minted a new class "expression of genes IRX3 and IRX5" that is a subclass of "gene expression" (Q26972) from Wikidata and is related to the class "IRX5" (Q18035174) and "IRX3" (Q18046058) from Wikidata.

\section{RDF Code}

This is our formalization as a nanopublication in TriG format:

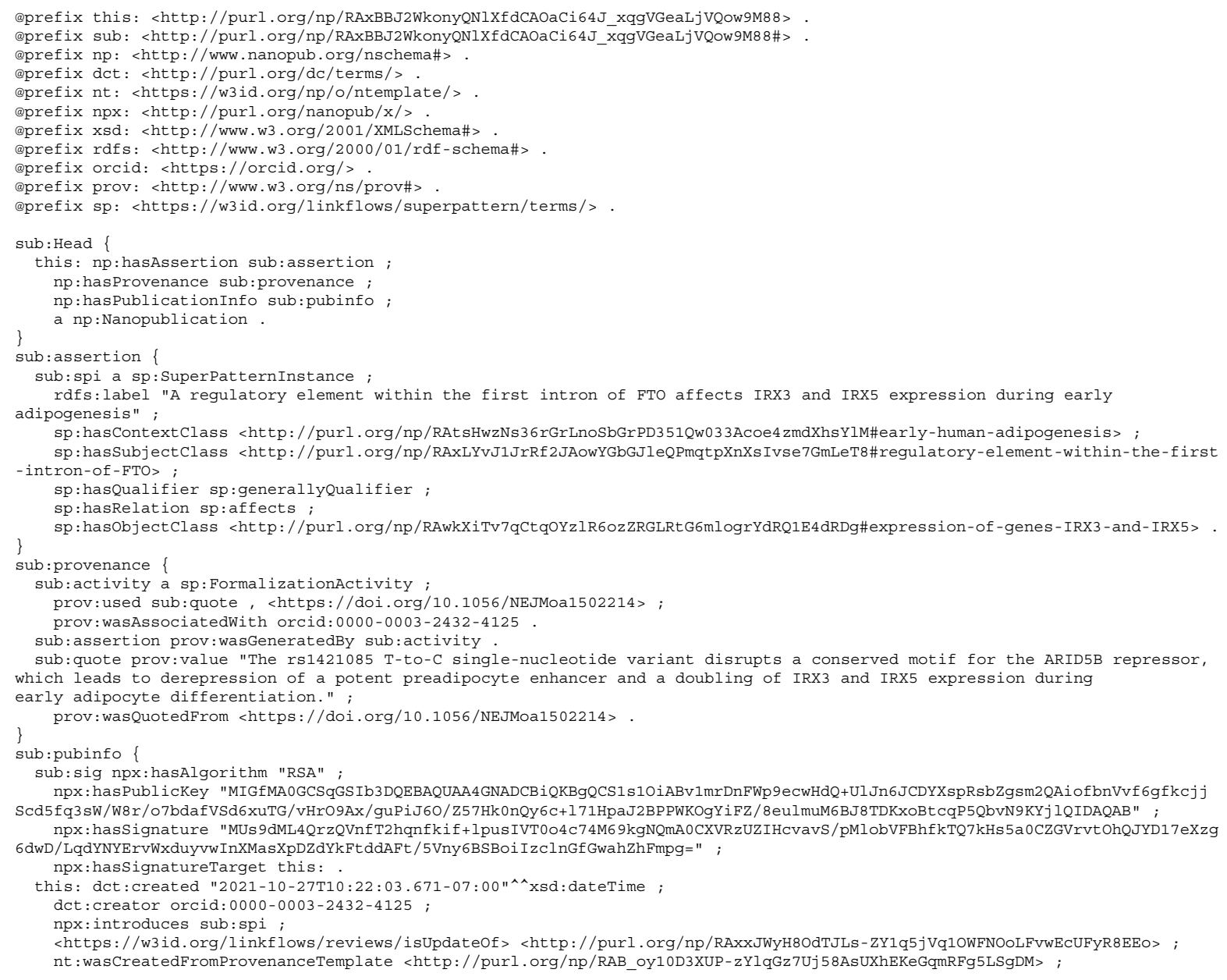


nt: wasCreatedFromPubinfoTemplate <http://purl.org/np/RAA2MfqdBCzmz9yVWj KLXNbyfBNCwSMmOqCNUxkk1maIM> , <http://purl.org/np/RAOGu9Lh0BD4tbIRB9RG6RGRA_ObDh75NTbIqaWgxxs8M>

$\mathrm{nt}$ : wasCreatedFromTemplate <http://purl.org/np/RAv68imZrEjfcp2rnEg1hzoBqEVc0ceMtp9_1Za0BxNM4>.

The following nanopublications introduce the newly minted classes in TriG format. This is the class definition of "early human adipogenesis":

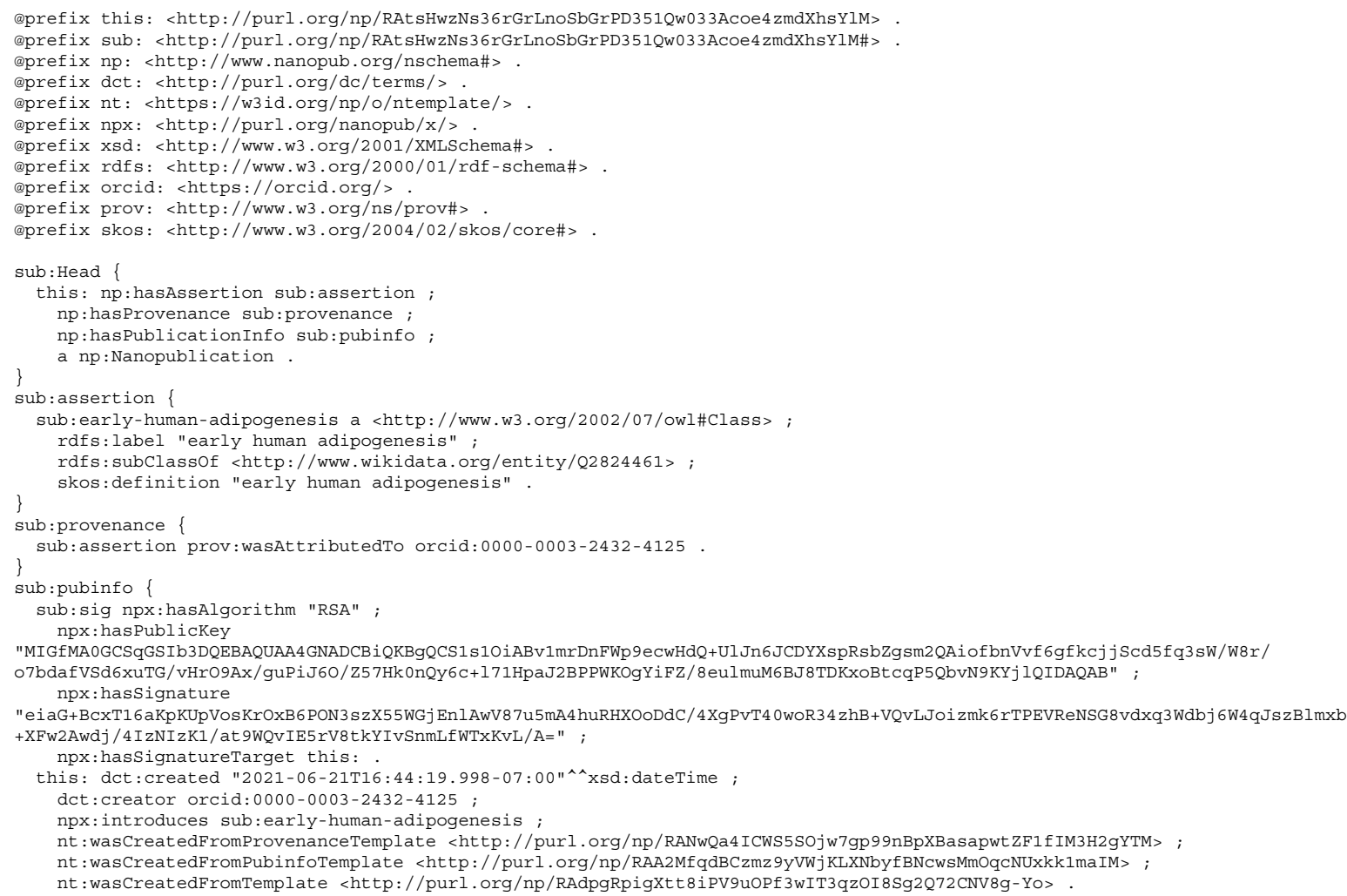

\section{This is the class definition of "regulatory element within the first intron of FTO":}

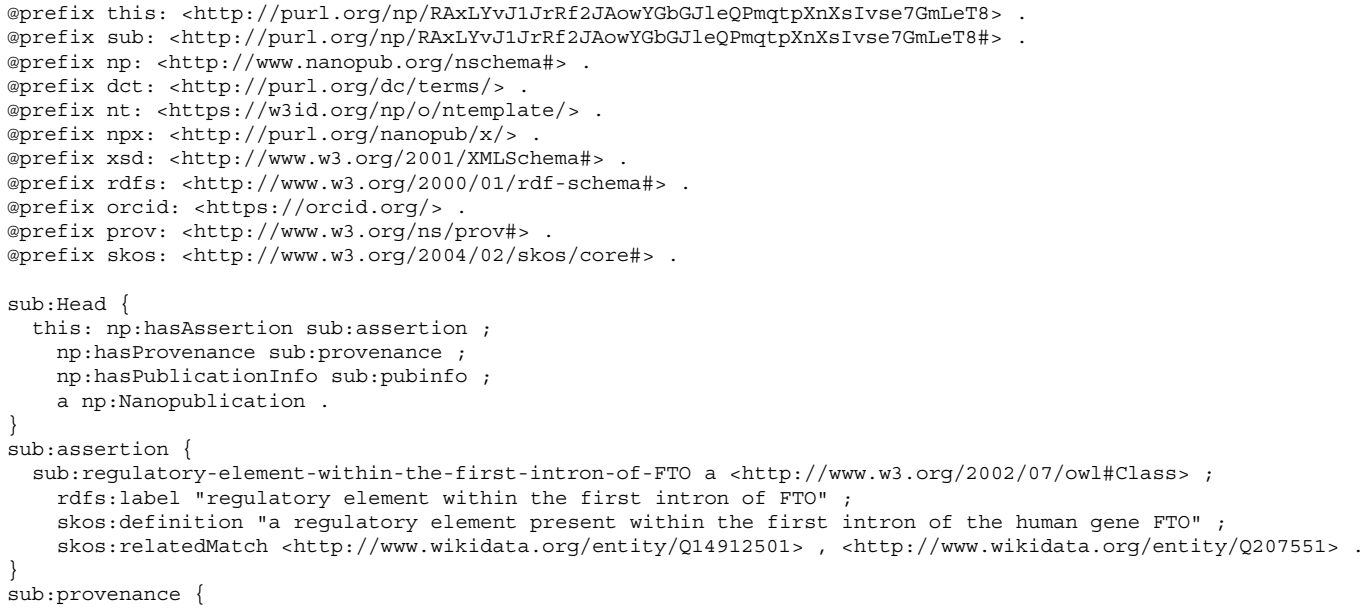


\}

sub:assertion prov: wasAttributedTo orcid:0000-0003-2432-4125.

sub: pubinfo \{

sub:sig npx:hasAlgorithm "RSA" ;

npx: hasPublickey

"MIGfMA0GCSqGSIb3DQEBAQUAA4 GNADCBiQKBgQCS1s10iABv1mrDnFWp9ecwHdQ+UlJn6JCDYXspRsbZgsm2QAiofbnVvf6gfkcjjjScd5fq3sW/W8r/

07bdafVSd6xuTG/vHr09Ax/guPiJ60/257Hk0nQY6c+171HpaJ2BPPWKOgYiFZ/8eulmuM6BJ8TDKxoBtcqP5QbvN9KYj1QIDAQAB" ; npx: hassignature

"M8CVwUkflLAuLqLUM+ul 7EdLSXQhcSsbERJUcBwTF2Ri 78XD6JNdHsF5+Qekg3xefqY7d8530m2RHPxjSkooNi3XAOZWsd2T60ZiZ01SmAIsaRarmsnHb1/ GDf00S4OiqCL1jVFNXMosdVd9runl tov655 znbpiFBJxmMd66+v0="

npx: hassignatureTarget this:

this: dct:created "2021-10-27T10:04:01.217-07:00"^^xsd:dateTime

dct: creator orcid:0000-0003-2432-4125;

npx:introduces sub:regulatory-element-within-the-first-intron-of-FTO

npx: supersedes <http://purl.org/np/RANAxL20xGmP9VAN6cUi OKEKOrJb9bPn4l1A5HguxKrI>

<https://w3id.org/linkflows/reviews/isUpdateof> <http://purl.org/np/RANAxL2OxGmP9VAN6cUi_0KEK0rJb9bPn4l1A5HquXKrI> ;

nt:wasCreatedFromProvenanceTemplate <http://purl.org/np/RANwQa4ICWS5SOjw7gp99nBpXBasapwtZF1fIM3H2gYTM> ;

nt: wasCreatedFromPubinfoTemplate <http://purl.org/np/RAA2MfqdBCzmz9yVWj KLXNbyfBNCWSMmOqCNUxkk1maIM> ,

<http://purl.org/np/RAOGu9Lh0BD4tbIRB9RG6RGRA_ObDh75NTbIqaWgxxs8M> ,

$<$ http://purl.org/np/RAjpBMlw3OwYhJUBo3DtsuDlXsNAJ8cnGeWAutDVjuAuI > ;

nt: wasCreatedFromTemplate <http://purl org/np/RAdpgRpigXtt8iPV9uOPf3wIT3qzOI8Sg2Q72CNV8g-Yo> .

This is the class definition of "expression of genes IRX3 and IRX5":

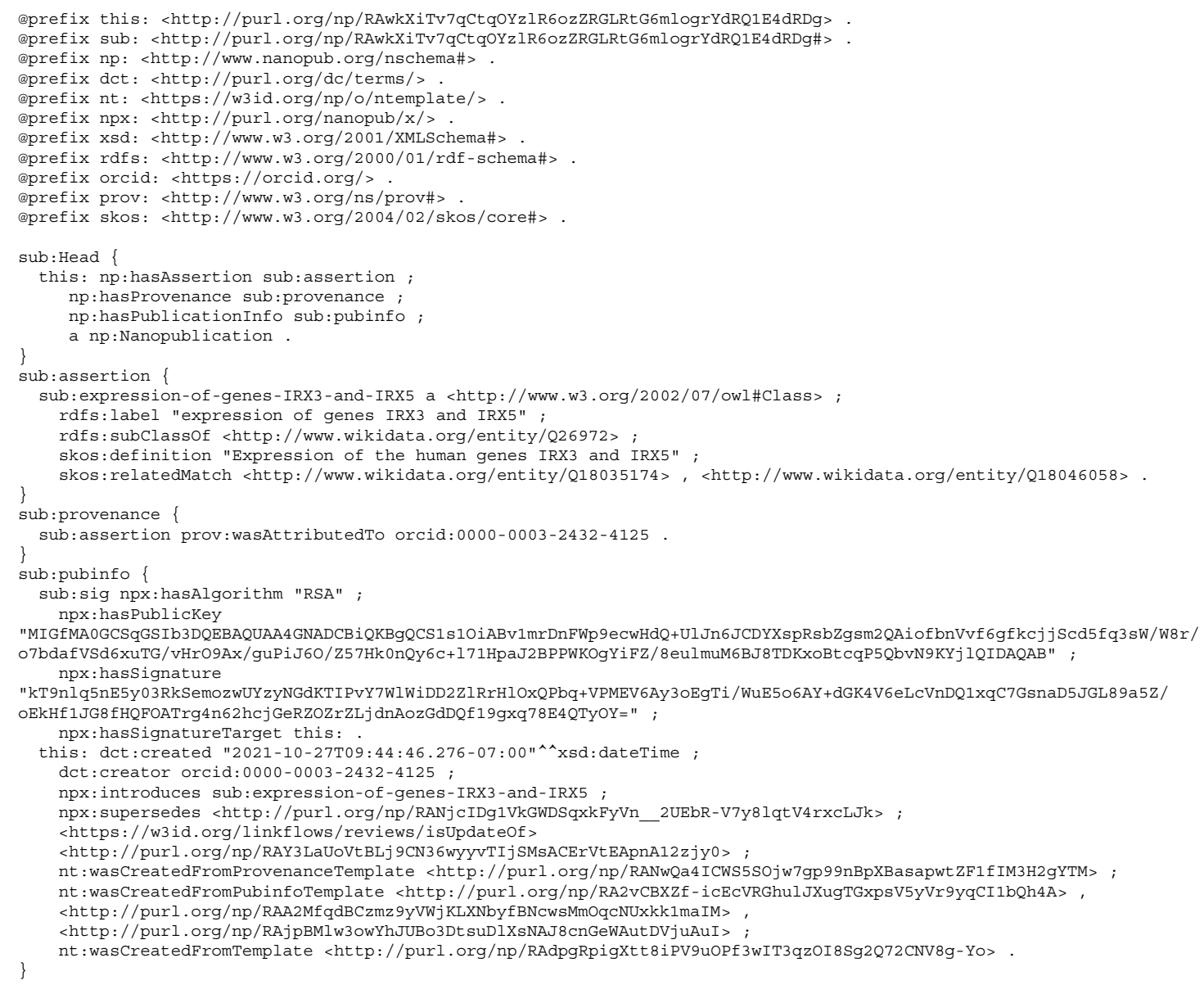

\section{References}

[1] M. Claussnitzer, S.N. Dankel, K.-H. Kim, G. Quon, W. Meuleman, C. Haugen, V. Glunk, I.S. Sousa, J.L. Beaudry, V. Puvi- 
indran, N.A. Abdennur, J. Liu, P.-A. Svensson, Y.-H. Hsu, D.J. Drucker, G. Mellgren, C.-C. Hui, H. Hauner and M. Kellis, FTO Obesity Variant Circuitry and Adipocyte Browning in Humans, New England Journal of Medicine 373(10) (2015), 895-907. doi:10.1056/NEJMoa1502214.

[2] C.I. Bucur, T. Kuhn, D. Ceolin and J. van Ossenbruggen, Expressing high-level scientific claims with formal semantics, in: Proceedings of the 11th Knowledge Capture Conference 2021. doi:10.1145/3460210.3493561. 